\title{
Genomic and Clinical Characterization of Stromal Infiltration Markers in Prostate Cancer
}

\author{
Brandon A. Mahal, MD (iD) 1; Mohammed Alshalalfa, PhD',2; Shuang G. Zhao, MD³; Himisha Beltran, MD'; \\ William S. Chen, MD (iD) 2; Fallon Chipidza, MD'; Elai Davicioni, PhD'; R. Jeffrey Karnes, MD5; Sheng-Yu Ku, PhD'; \\ Tamara L. Lotan, MD ${ }^{6}$; Vinayak Muralidhar, MD'; Timothy R. Rebbeck, PhD ${ }^{1,7}$; Edward M. Schaeffer, MD ${ }^{8}$; \\ Daniel E. Spratt, MD (iD) ${ }^{3}$; Felix Y. Feng, MD²; and Paul L. Nguyen, MD ${ }^{1}$
}

\begin{abstract}
BACKGROUND: The progression of prostate cancer is a complex, multistep process that involves molecular alterations in cells of the tumor and the microenvironment, with associated interactions between the stroma and epithelium. Genomic expression analyses of stromal infiltration markers were performed to determine the significance thereof in prostate cancer. METHODS: Genome-wide expression profiles of formalin-fixed, paraffin-embedded radical prostatectomy samples were evaluated from a prospective registry cohort $(n=5239)$ and 3 retrospective institutional cohorts $(n=1135)$. Two independent stromal gene expression signatures implied stromal infiltration. Cox proportional hazards regression defined the association between stromal infiltration expression and metastasis-free survival (MFS). RESULTS: Stromal expression scores were correlated with stromal signature genes and with other key stromal markers (CAV1, VIM, and TAGLN), basal activity, and CD3 and CD4 immune biomarkers ( $r>0.5$ for all). The top decile of stromal expression was associated with high genomic risk scores (Decipher $\geq 0.6$ ), high Cancer of the Prostate Risk Assessment-Postsurgical scores, Gleason 9 to 10 disease, and a higher risk for metastasis (hazard ratio, 2.35; $95 \% \mathrm{Cl}, 1.37-4.02 ; P=.001$ ). A higher stromal infiltration score was also associated with decreased expression of DNA repair genes and higher radiation sensitivity genomic scores. Postoperative radiation therapy (RT) was associated with an MFS benefit for patients with high stromal scores, but not for patients with low stromal scores $\left(P_{\text {interaction }}=.02\right)$. CONCLUSIONS: Expression of stromal infiltration markers is correlated with prostate cancer aggressiveness/progression and may be predictive of a response to RT. Stromal infiltration markers should be studied and considered for incorporation into clinical prognostication and decision making. Cancer 2020;126:1407-1412. (c) 2020 American Cancer Society.
\end{abstract}

KEYWORDS: Decipher, genomics, prostatic neoplasms, stromal infiltration, tumor microenvironment.

\section{INTRODUCTION}

Prostate cancer is the most commonly diagnosed nonskin cancer in men. ${ }^{1}$ Prognostication and treatment decisions have been guided by tumor stage, prostate cancer-specific antigen, and Gleason score for the last several decades. ${ }^{2}$ Nevertheless, the progression of prostate cancer is a complex, multistep process that involves molecular alterations in cells of the tumor and the microenvironment, with associated interactions between the stroma and epithelium that cannot be entirely explained by clinical factor risk criteria alone. ${ }^{3}$

Genomics in prostate cancer has led to a more in-depth investigation and understanding of molecular alterations in cells of the tumor and the microenvironment. ${ }^{4-6}$ As such, genomics is increasingly being incorporated into prognostication, treatment decisions, and targeted therapy design for prostate cancer. Still, the clinical significance and implications of stromal infiltration in primary prostate cancer are not well-defined or understood. ${ }^{3}$

Therefore, we performed genomic expression analyses of stromal infiltration markers and sought to determine the clinical significance thereof in prostate cancer.

\section{MATERIALS AND METHODS}

\section{Study Cohorts}

Genome-wide expression profiles of formalin-fixed, paraffin-embedded (FFPE) radical prostatectomy (RP) tumor samples, from patients with clinically localized prostate cancer, were evaluated from a prospective registry cohort $(\mathrm{n}=5239)$

Corresponding Author: Brandon A. Mahal, MD, McGraw/Patterson Center for Population Sciences, Dana-Farber Cancer Institute, 450 Brookline Ave, 1101 Dana, Boston, MA 02215 (brandon_mahal@dfci.harvard.edu).

${ }^{1}$ Dana-Farber Cancer Institute and Brigham and Women's Hospital, Boston, Massachusetts; ${ }^{2}$ University of California at San Francisco, San Francisco, California; ${ }^{3}$ University of Michigan, Ann Arbor, Michigan; ${ }^{4}$ GenomeDx, Inc, San Diego, California; ${ }^{5}$ Mayo Clinic, Rochester, Minnesota; ${ }^{6}$ Johns Hopkins Medical Institutions, Baltimore, Maryland; ${ }^{7}$ Harvard T.H. Chan School of Public Health, Boston, Massachusetts; ${ }^{8}$ Northwestern University, Chicago, Illinois

The first 2 authors contributed equally to this article.

The last 2 authors contributed equally to this article.

Additional supporting information may be found in the online version of this article.

DOI: 10.1002/cncr.32688, Received: September 7, 2019; Revised: November 5, 2019; Accepted: December 4, 2019, Published online January 6, 2020 in Wiley Online Library (wileyonlinelibrary.com) 
and 3 retrospective institutional cohorts $(\mathrm{n}=1135)$. The Cancer Genome Atlas (TCGA) prostate cohort was used for validation across platforms $(n=498){ }^{7}$ The prospective cohort was composed of anonymized genome-wide expression profiles from clinical use of the Decipher test between February 2014 and August 2016 retrieved from the Decipher Genomics Resource Information Database (GRID; NCT02609269), and basic demographic and pathological data were included. The retrospective cohorts included patients treated with RP at Johns Hopkins University $(\mathrm{n}=355)$ and the Mayo Clinic (I, $\mathrm{n}=545$; II, $\mathrm{n}=235$ ) and included adequate follow-up for the endpoint of metastasis-free survival (MFS). ${ }^{8-10}$ Supporting Figure 1 summarizes the patient cohorts in a flow diagram.

A central pathology review was performed for all cases. Before tissue sampling for the clinical Decipher assay, a histologic review of the submitted FFPE block was performed by a pathologist. Details regarding pathology procedures, including microarray preprocessing and normalization, have been previously described. ${ }^{11-13}$ Notably, an attempt was made to identify all available FFPE blocks (including lymph node blocks); the block containing the dominant Gleason tumor was selected for RNA isoloation. ${ }^{11}$ From there, freshly cut sections from the FFPE blocks (four $10-\mu \mathrm{m}$ sections) were deparaffinized before macrodissection of the dominant Gleason tumor for RNA extraction. The acceptance criteria for the Decipher assay included at least $0.5 \mathrm{~cm}^{2}$ of tumor with at least $60 \%$ neoplastic cells. Details regarding RNA extraction and laboratory methods have been previously described. ${ }^{4}$

\section{Statistical Analysis}

We used the Estimation of Stromal and Immune Cells in Malignant Tumor Tissues Using Expression Data (ESTIMATE) algorithm of 141 stromal genes to infer stromal infiltration from gene expression data. ${ }^{14}$ In addition, we used a 27 -gene stromal signature ${ }^{3}$ with overlap of 9 genes from the 141-gene signature. For the TCGA prostate adenocarcinoma cohort, we downloaded the ESTIMATE stromal scores, immunohistochemistry (IHC), and consensus measurement of purity estimates scores. ${ }^{7}$ ERGfusion frequency was examined across deciles of stromal scores as a proxy for tumor purity/tumor signal. ${ }^{15}$

The distribution of high genomic risk for metastasis (Decipher score $\geq 0.6$ ), high Cancer of the Prostate Risk Assessment-Postsurgical (CAPRA-S) scores, and Gleason 9 to 10 disease across deciles of stromal infiltration expression was assessed.

Cox proportional hazards regression defined the association between stromal infiltration expression (high [top decile] vs low) and MFS (metastases defined by radiographic evidence) after RP; a multivariable analysis was also performed with adjustments for Gleason score to evaluate the association of stromal infiltration expression and MFS, independent of Gleason score. Lastly, associations between stromal infiltration and radiation response scores were tested with a 24-gene radiation sensitivity signature (Post-Operative Radiation Therapy Outcomes Score $[\text { PORTOS] })^{12}$ and an IFN-related DNA damage resistance signature. Cox proportional hazards were used to examine the association between stromal infiltration (high [top decile] vs low) and MFS by the receipt of post-RP radiation therapy (RT), including a stromal infiltration $\times \mathrm{RT}$ interaction term, in a previously published matched cohort $(n=196$; half of the patients received post-RP RT); this cohort was specifically matched (exactly 1:1) on preoperative prostate-specific antigen levels, surgical Gleason scores, surgical margin status, extracapsular extension, seminal vesicle invasion, lymph node invasion, and androgen deprivation therapy. ${ }^{12}$

Spearman's correlation was used for correlation analysis. Statistical analyses were performed with $\mathrm{R}$ v3.3.1, and a 5\% significance level was applied for all tests. Local institutional review boards approved all data collection.

\section{RESULTS}

\section{Baseline Characteristics}

In the prospective $(\mathrm{n}=5239)$ and retrospective cohorts $(\mathrm{n}=1135)$, at diagnosis, the median ages were 65 and 64 years, the median prostate-specific antigen levels were 6.6 and $9.0 \mathrm{ng} / \mathrm{mL}$, and $18 \%$ and $43 \%$ of the patients had Gleason scores of 8 to 10 , respectively.

\section{Distribution of Stromal Genomic Expression}

In the prospective cohort, there was a strong correlation between stromal expression scores (based on the ESTIMATE algorithm) and the 141 genes composing that signature (Supporting Fig. 2A). The stromal score was strongly correlated with other key well-established stromal markers (genes) not included in the 141-gene stromal signature (CAV1 $[r=0.59]$, VIM $[r=0.74]$, TAGLN $[r=0.62]$, and CNN1 $[r=0.6])$, basal activity $(r=0.72)$, and CD3 $(r=0.45)$ and CD4 $(r=0.5)$ immune biomarkers and with another independent stromal score based on 27 genes $(r=0.84$; Supporting Fig. 2B). Furthermore, ERG-fusion frequency was similar across deciles of stromal scores (Supporting Fig. 2C). Because IHC data for stromal markers were not available in the GRID data, IHC data and consensus purity estimates were used from 
the TCGA prostate adenocarcinoma cohort; ${ }^{7}$ Both tumor purity measures were negatively associated with the stromal score, and this indicated that the stromal score reflected stromal infiltration (Supporting Fig. 2D,E). Stromal expression scores were similar across Gleason scores and genomic risk (Decipher) scores (Supporting Fig. 3).

\section{Outcomes by Stromal Genomic Expression}

The top decile of stromal expression was associated with high genomic risk for metastasis (Decipher $\geq 0.6$ ), high CAPRA-S scores, and Gleason 9 to 10 disease $(P<.05$ for all [Mann-Kendall trend test]; Fig. 1A-C). The distribution of stromal expression across Gleason and genomic risk (Decipher) scores is displayed in Supporting Figure 3; notably, $41 \%$ of Gleason $3+3$ tumors, $47 \%$ of Gleason $3+4$ tumors, and $48 \%$ of Gleason $4+3$ tumors had stromal expression scores above the median, and $36 \%$ of Gleason 9 to 10 tumors had stromal expression scores below the median. The top decile of stromal expression (compared with lower stromal expression) was associated with a higher risk of metastasis in the Johns Hopkins University (hazard ratio [HR], 2.35; 95\% CI, 1.37-4.02; $P=.001)$ and Mayo Clinic cohorts (HR, 1.38; 95\% CI, 1.02-1.86; $P=.04$; Fig. $1 \mathrm{D}, \mathrm{E})$; there was a higher but nonsignificant risk of disease progression in the TCGA cohort (HR, 1.82; 95\% CI, 0.94-3.50; $P=.06$; Fig. 1F). In a multivariable analysis with adjustments for Gleason score, the top decile of stromal scores remained independently associated with a higher risk of metastasis (adjusted HR, 2.15; 95\% CI, 1.25-3.7; $P=.005$ ).

Furthermore, the stromal score was correlated with the radiation sensitivity PORTOS score $(r=0.37)$, and a high PORTOS score $(>0)$ was associated with higher stromal infiltration $(P<.001$; Fig. $2 \mathrm{~A})$. The stromal score was also negatively correlated with DNA repair activity $(r=-0.75$; Fig. 2B). In a matched cohort of patients treated with postoperative RT $(n=98)$ versus not $(n=98)$, postoperative RT was associated with an MFS benefit for patients with high (top-decile) stromal scores but not for patients with low stromal scores $\left(P_{\text {interaction }}=.02\right.$; Fig. 2C-E); 10-year MFS rates for high versus low stromal scores were $24 \%$ versus $68 \%(P=.0015)$ and $50 \%$ versus $54 \%(P=.45)$ for patients who did not versus who did receive postoperative RT, respectively.

\section{DISCUSSION}

This study highlights the novel finding that the expression of stromal infiltration markers is correlated with prostate cancer aggressiveness/progression and may be predictive of a response to RT. Specifically, high expression of stromal infiltration markers was associated with a highrisk Decipher genomic risk score $(\geq 0.6)$, a high CAPRA-S score, Gleason 9 to 10 disease, and a higher risk of metastases after RP. Lastly, higher expression of stromal infiltration was associated with high radiation sensitivity genomic scores, low DNA repair activity, and improved MFS with RT. There was an interaction between high stromal expression and the receipt of RT such that the significant MFS benefit of RT was limited to patients with high stromal expression. To our knowledge, this study includes the first data to demonstrate such findings.

Together, these results suggest that stromal infiltration marker expression may be both prognostic and predictive in prostate cancer. Notably, although high expression of stromal infiltration markers was associated with a high Gleason score, stromal expression was prognostic for the risk of metastasis independent of Gleason score in the multivariable Cox regression analysis. As such, the expression of stromal microenvironment markers may have an important independent role in predicting the risk of adverse events in prostate cancer. Furthermore, whether higher expression of stromal infiltration markers is associated with a better response to RT needs further exploration in studies with long clinical follow-up.

Because infiltrating stromal cells and other immune cells account for a majority of "normal" cells found in solid tumor tissues, these findings have important, clinically relevant implications. The mechanisms of prostate cancer development and progression are a complex process that involves alterations of the tumor and microenvironment, where stromal cells likely affect disease progression and treatment response. ${ }^{3}$ At present, prognostic tools for prostate cancer are principally based on information provided by tumor cells (eg, the Gleason score, size of tumor, or tumor genomics). ${ }^{16,17}$ However, increasing evidence suggests that stromal and immune cells are critical for disease progression and drug resistance. ${ }^{18-20}$

The infiltration of stromal and microenvironment cells may influence genomic or gene expression approaches to prognostic and predictive models because of the implications for tumor heterogeneity and purity. The ESTIMATE method uses gene expression data to infer the fractional content of stromal and immune cells in tumor samples, and this allows for a straightforward approach to assessing tumor purity and stromal infiltration in tumor samples by the use of gene expression data. ${ }^{14}$ Therefore, stromal expression scores can help to inform tumor purity/heterogeneity estimates by assessing for the presence of stromal infiltration. Furthermore, the findings in this study suggest that levels of stromal infiltration 


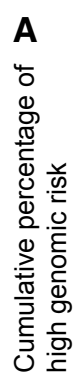

5,239 RP samples (Decipher GRID)

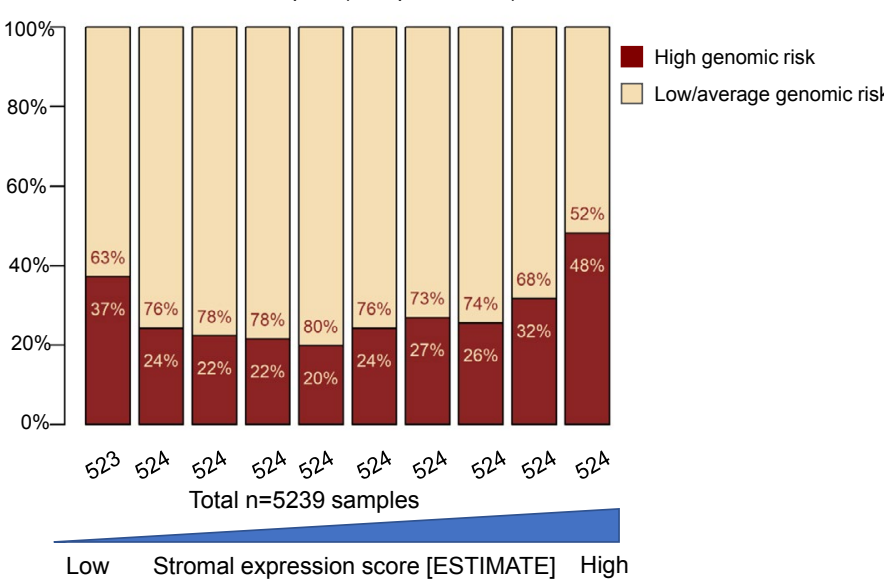

D
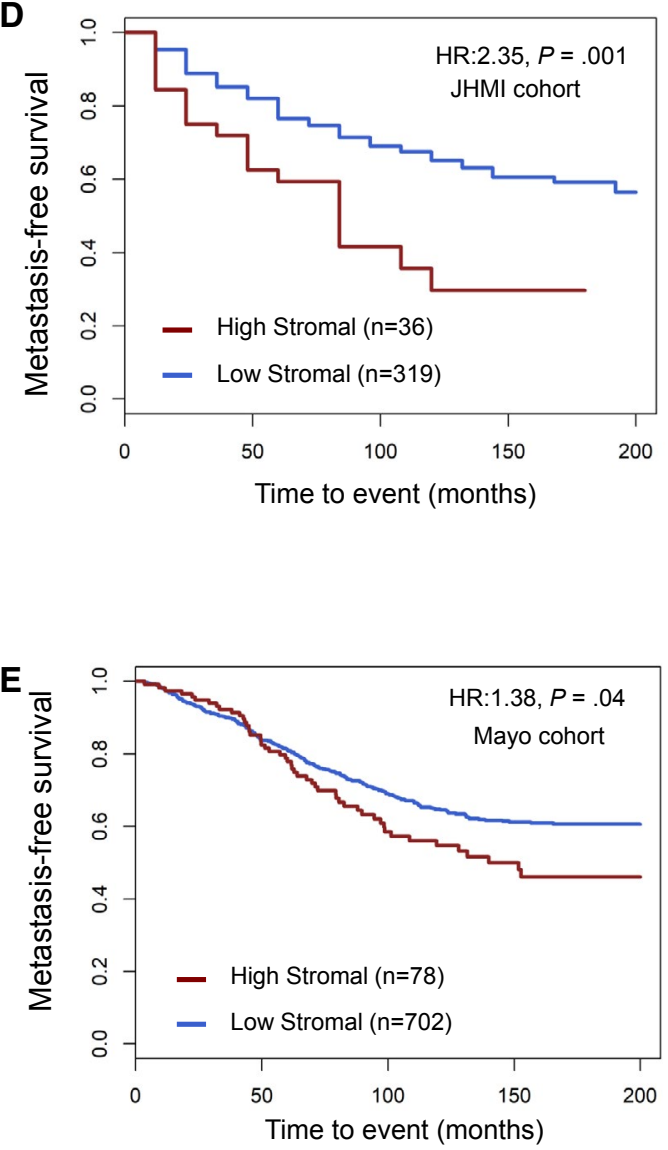

$\mathbf{F}$

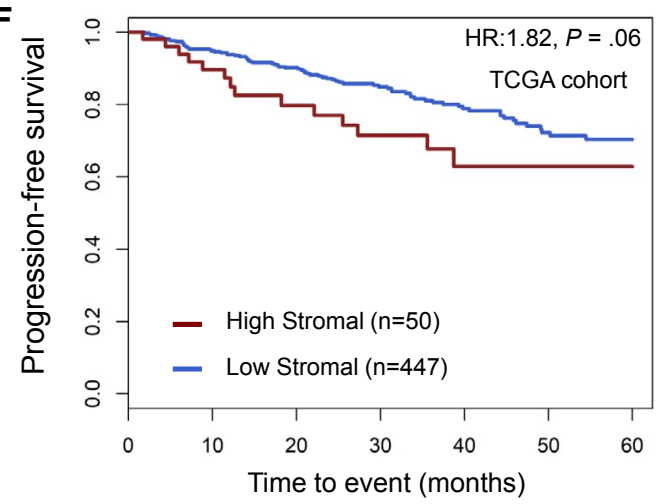

Figure 1. Distribution of (A) high genomic risk scores (Decipher scores $\geq 0.6$ ), (B) high CAPRA-S scores, and (C) Gleason 9 to 10 disease, across deciles of stromal expression scores $(P<.05$ [Mann-Kendall trend test]). Survival analyses stratified by stromal scores (high $=$ top decile) of (D,E) metastasis-free survival over time after RP in the Johns Hopkins University and Mayo Clinic cohorts, respectively, and (F) progression-free survival in the TCGA cohort. CAPRA-S indicates Cancer of the Prostate Risk Assessment-Postsurgical; ESTIMATE, Estimation of Stromal and Immune Cells in Malignant Tumor Tissues Using Expression Data; GRID, Genomics Resource Information Database; GS, Gleason score; HR, hazard ratio; JHMI, Johns Hopkins Medical Institute; RP, radical prostatectomy; TCGA, The Cancer Genome Atlas. 

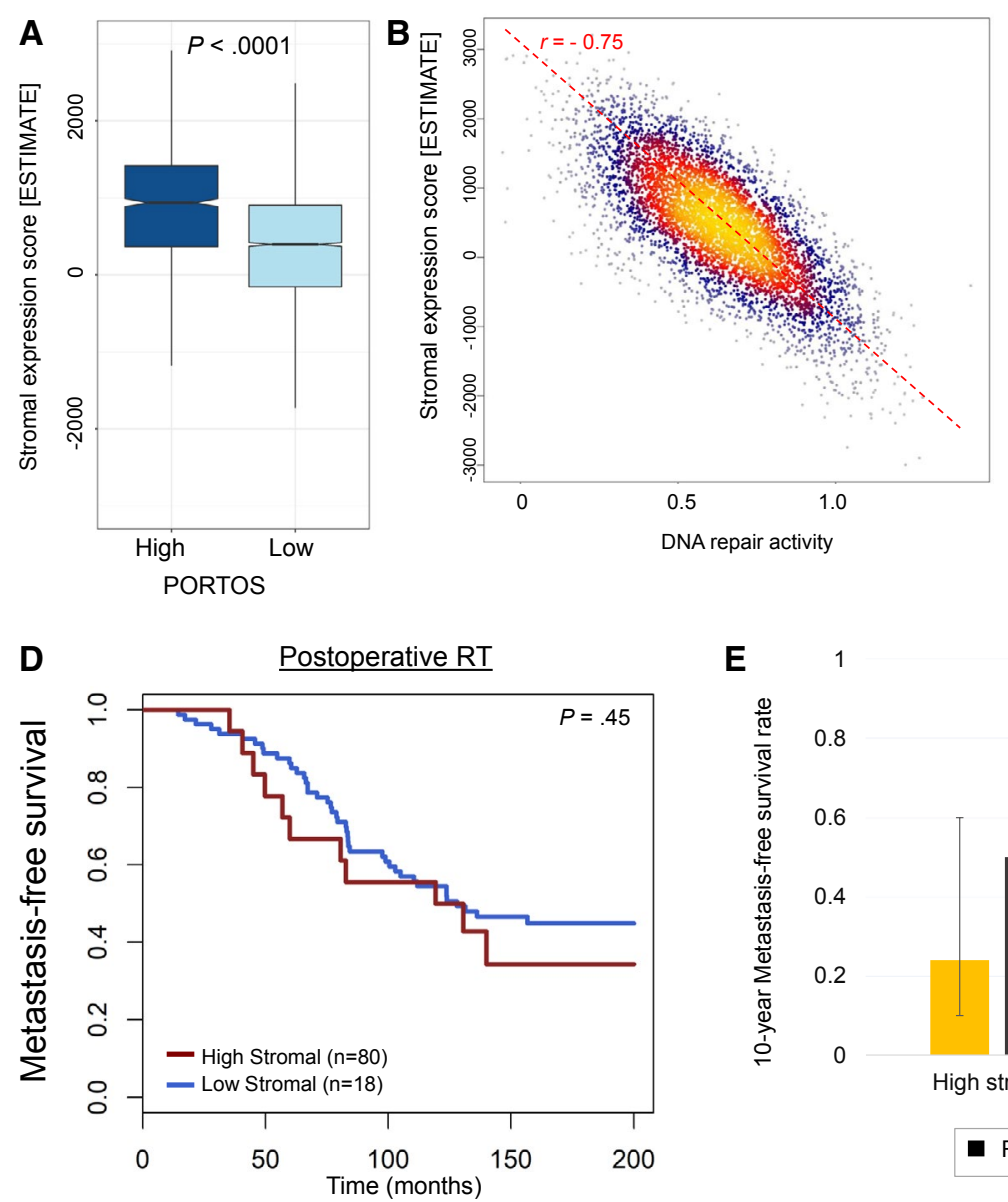

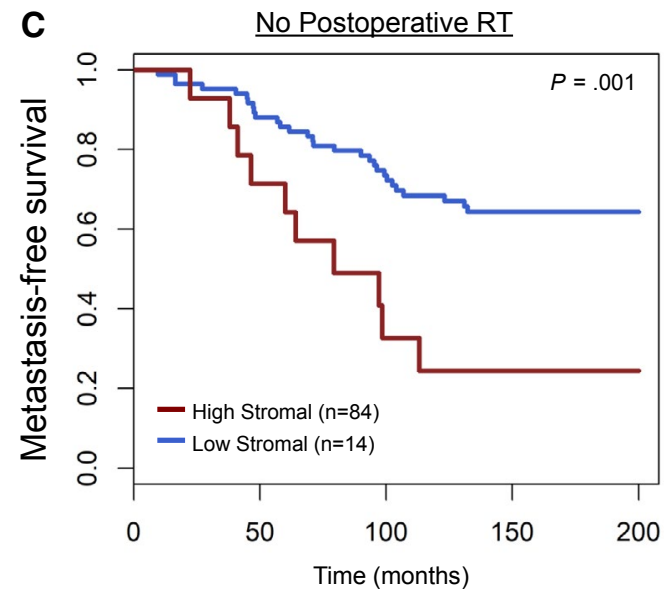

Figure 2. Association between stromal expression scores and (A) a 24-gene radiation sensitivity signature (PORTOS), (B) a DNA repair activity signature, and (C-E) metastasis-free survival by high stromal expression (top decile) versus low stromal expression and receipt of post-radical prostatectomy RT. ESTIMATE indicates Estimation of Stromal and Immune Cells in Malignant Tumor Tissues Using Expression Data; PORTOS, Post-Operative Radiation Therapy Outcomes Score; RT, radiation therapy.

are likely associated with clinical characteristics and outcomes. With the ongoing shift toward the incorporation of genomics into prognostication and trial design for prostate and other cancers, stromal infiltration and other tumor microenvironment markers must be considered.

The major limitations of this study include the lack of long-term clinical follow-up for the prospective cohort to allow for clinical analyses and the inherent limitations of retrospective analyses in the clinical findings. Moreover, the study was limited by a lack of IHC-based stromal quantification for samples from the Decipher cohort. Nevertheless, the clinical analyses were explored in multiple independent retrospective cohorts, and ERG+ distribution and purity analyses support a strong tumor signal in the findings. Furthermore, the distribution of stromal scores across well-established prostate cancer risk factors suggests possible nonmonotonic behavior in which a low stromal score may also represent an adverse feature; however, this study may be underpowered to detect such differences.

Ultimately, stromal infiltration markers should be further investigated and considered for incorporation into clinical trials and ultimately clinical prognostication and treatment decision making.

In conclusion, despite any potential limitations, this study demonstrated the novel finding that high genomic expression of stromal infiltration markers was associated with aggressive disease, adverse prostate cancer outcomes, and a better response to RT. Stromal infiltration markers should be considered for incorporation into clinical prognostication and treatment decision making.

\section{FUNDING SUPPORT}

Brandon A. Mahal is funded by the Prostate Cancer Foundation/American Society for Radiation Oncology Award to End Prostate Cancer (grant 
support). Timothy R. Rebbeck has reported the receipt of grants from the National Institutes of Health. Paul L. Nguyen is funded by the Prostate Cancer Foundation (grant support). This work was also supported by the Wood Family Foundation, the Baker family, the Freedman family, Fitz's Cancer Warriors, David and Cynthia Chapin, the Frashure family, Hugh Simons in honor of Frank and Anne Simons, the Campbell family in honor of Joan Campbell, the Scott Forbes and Gina Ventre Fund, and a grant from an anonymous family foundation (grant support).

\section{CONFLICT OF INTEREST DISCLOSURES}

Mohammed Alshalalfa reports personal fees from GenomeDx outside the submitted work. Brandon Mahal reports grants from the Department of Defense, Prostate Cancer Foundation, and American Society for Radiation Oncology; and travel honoraria from Genzyme, the Exeter Group, and the Prostate Health Education Network outside the submitted work. Shuang G. Zhao reports nonfinancial support and other from GenomeDx Biosciences outside the submitted work; in addition, Zhao reports 3 patents pending to GenomeDx Biosciences. Elai Davicioni reports personal fees from GenomeDx (Decipher) outside the submitted work. R. Jeffrey Karnes reports royalties from GenomeDx outside the submitted work. Tamara L. Lotan reports grants from the Prostate Cancer Foundation outside the submitted work. Daniel E. Spratt reports grants from the Prostate Cancer Foundation and personal fees from Janssen, Blue Earth, and AstraZeneca outside the submitted work. Felix Y. Feng reports personal fees from Dendreon, EMD Serono, Janssen Oncology, Ferring, Sanofi, Bayer, Blue Earth Diagnostics, Celgene, Medivation/Astellas, and Clovis Oncology and ownership interests in PFS Genomics outside the submitted work; in addition, Feng has a patent issued (EP3047037 A4). Paul L. Nguyen reports the receipt of consulting fees from Ferring, Bayer, Janssen, Astellas, Dendreon, GenomeDx, Blue Earth Diagnostics, Cota, Boston Scientific, and Nanobiotix; grant funding from Janssen, Bayer, and Astellas; and equity in Augmenix. The other authors made no disclosures.

\section{AUTHOR CONTRIBUTIONS}

Brandon A. Mahal: Data access, responsibility, and analysis; statistical analysis; concept and design; acquisition, analysis, and interpretation of data; drafting of the manuscript; and critical revision of the manuscript for important intellectual content. Mohammed Alshalalfa: Data access, responsibility, and analysis; statistical analysis; concept and design; acquisition, analysis, and interpretation of data; drafting of the manuscript; and critical revision of the manuscript for important intellectual content. Shuang G. Zhao: Concept and design; acquisition, analysis, and interpretation of data; drafting of the manuscript; and critical revision of the manuscript for important intellectual content. Himisha Beltran: Concept and design; acquisition, analysis, and interpretation of data; drafting of the manuscript; and critical revision of the manuscript for important intellectual content. William S. Chen: Concept and design; acquisition, analysis, and interpretation of data; drafting of the manuscript; and critical revision of the manuscript for important intellectual content. Fallon Chipidza: Concept and design; acquisition, analysis, and interpretation of data; drafting of the manuscript; and critical revision of the manuscript for important intellectual content. Elai Davicioni: Concept and design; acquisition, analysis, and interpretation of data; drafting of the manuscript; and critical revision of the manuscript for important intellectual content. R. Jeffrey Karnes: Concept and design; acquisition, analysis, and interpretation of data; drafting of the manuscript; and critical revision of the manuscript for important intellectual content. Sheng-Yu Ku: Concept and design; acquisition, analysis, and interpretation of data; drafting of the manuscript; and critical revision of the manuscript for important intellectual content. Tamara L. Lotan: Concept and design; acquisition, analysis, and interpretation of data; drafting of the manuscript; and critical revision of the manuscript for important intellectual content. Vinayak Muralidhar: Concept and design; acquisition, analysis, and interpretation of data; drafting of the manuscript; and critical revision of the manuscript for important intellectual content. Timothy $\mathbf{R}$. Rebbeck: Concept and design; acquisition, analysis, and interpretation of data; drafting of the manuscript; and critical revision of the manuscript for important intellectual content. Edward M. Schaeffer: Concept and design; acquisition, analysis, and interpretation of data; drafting of the manuscript; and critical revision of the manuscript for important intellectual content. Daniel E. Spratt: Concept and design; acquisition, analysis, and interpretation of data; drafting of the manuscript; and critical revision of the manuscript for important intellectual content. Felix Y. Feng: Concept and design; acquisition, analysis, and interpretation of data; drafting of the manuscript; and critical revision of the manuscript for important intellectual content. Paul L. Nguyen: Concept and design; acquisition, analysis, and interpretation of data; drafting of the manuscript; and critical revision of the manuscript for important intellectual content.

\section{REFERENCES}

1. Siegel RL, Miller KD, Jemal A. Cancer statistics, 2019. CA Cancer J Clin. 2019;69:7-34.

2. D'Amico AV, Whittington R, Malkowicz SB, et al. Biochemical outcome after radical prostatectomy, external beam radiation therapy, or interstitial radiation therapy for clinically localized prostate cancer. JAMA. 1998;280:969-974.

3. Tyekucheva S, Bowden M, Bango C, et al. Stromal and epithelial transcriptional map of initiation progression and metastatic potential of human prostate cancer. Nat Commun. 2017;8:420.

4. Spratt DE, Yousefi K, Deheshi S, et al. Individual patient-level meta-analysis of the performance of the Decipher genomic classifier in high-risk men after prostatectomy to predict development of metastatic disease. J Clin Oncol. 2017;35:1991-1998.

5. Alshalalfa M, Nguyen PL, Beltran H, et al. Transcriptomic and clinical characterization of neuropeptide $\mathrm{Y}$ expression in localized and metastatic prostate cancer: identification of novel prostate cancer subtype with clinical implications. Eur Urol Oncol. 2019;2:405-412.

6. Mahal BA, Alshalalfa M, Spratt DE, et al. Prostate cancer genomic-risk differences between African-American and white men across Gleason scores. Eur Urol. 2019;75:1038-1040.

7. Aran D, Sirota M, Butte AJ. Systematic pan-cancer analysis of tumour purity. Nat Commun. 2015;6:8971.

8. Erho N, Crisan A, Vergara IA, et al. Discovery and validation of a prostate cancer genomic classifier that predicts early metastasis following radical prostatectomy. PLoS One. 2013;8:e66855.

9. Ross $\mathrm{AE}$, Johnson $\mathrm{MH}$, Yousefi $\mathrm{K}$, et al. Tissue-based genomics augments post-prostatectomy risk stratification in a natural history cohort of intermediate- and high-risk men. Eur Urol. 2016;69:157-165.

10. Karnes RJ, Bergstralh EJ, Davicioni E, et al. Validation of a genomic classifier that predicts metastasis following radical prostatectomy in an at risk patient population. J Urol. 2013;190:2047-2053.

11. Nakagawa T, Kollmeyer TM, Morlan BW, et al. A tissue biomarker panel predicting systemic progression after PSA recurrence post-definitive prostate cancer therapy. PLoS One. 2008;3:e2318.

12. Zhao SG, Chang SL, Spratt DE, et al. Development and validation of a 24-gene predictor of response to postoperative radiotherapy in prostate cancer: a matched, retrospective analysis. Lancet Oncol. 2016; $17: 1612-1620$.

13. Zhao SG, Chang SL, Erho N, et al. Associations of luminal and basal subtyping of prostate cancer with prognosis and response to androgen deprivation therapy. JAMA Oncol. 2017;3:1663-1672.

14. Yoshihara K, Shahmoradgoli M, Martinez E, et al. Inferring tumour purity and stromal and immune cell admixture from expression data. Nat Commun. 2013;4:2612.

15. Baca SC, Prandi D, Lawrence MS, et al. Punctuated evolution of prostate cancer genomes. Cell. 2013;153:666-677.

16. Muralidhar V, Zhang J, Wang Q, et al. Genomic validation of 3-tiered clinical sub-classification of high-risk prostate cancer. Int J Radiat Oncol Biol Phys. 2019;105:621-627.

17. Zhao SG, Chen WS, Das R, et al. Clinical and genomic implications of luminal and basal subtypes across carcinomas. Clin Cancer Res. 2019;25:2450-2457.

18. Kalluri R. The biology and function of fibroblasts in cancer. Nat Rev Cancer. 2016;16:582-598.

19. Straussman R, Morikawa T, Shee K, et al. Tumour micro-environment elicits innate resistance to RAF inhibitors through HGF secretion. Nature. 2012;487:500-504.

20. Hanahan D, Weinberg RA. Hallmarks of cancer: the next generation. Cell. 2011;144:646-674. 